\title{
Screening of candidate key genes associated with human osteosarcoma using bioinformatics analysis
}

\author{
KEFENG ZHANG, JIANWEN GAO and YONG NI \\ Department of Spinal Surgery, Shandong Jining No. 1 People's Hospital, Jining, Shandong 272011, P.R. China
}

Received October 20, 2015; Accepted March 23, 2017

DOI: 10.3892/ol.2017.6519

\begin{abstract}
The aim of the present study was to identify the key genes associated with osteosarcoma (OS) using a bioinformatics approach. Microarray data (GSE36004) was downloaded from the Gene Expression Omnibus database, including 19 OS cell lines and 6 normal controls. Differentially expressed genes (DEGs) in the OS cell lines were identified using the Limma package, and differentially methylated regions were screened with methyAnalysis in R. Copy number analysis was performed and genes with copy number gains/losses were further screened using DNAcopy and cghMCR packages. Functional enrichment analyses were performed using the Database for Annotation, Visualization and Integrated Discovery online tool, and protein-protein interactions were identified based on information obtained from the Search Tool for the Retrieval of Interacting Genes database. A total of 47 downregulated genes were screened in hyper-methylated regions, including the fragment crystallizable $(\mathrm{Fc})$ region of immunoglobulin $\mathrm{E}$, high affinity I, receptor for; $\gamma$ polypeptide $(F C E R I G)$, leptin $(L E P)$ and feline Gardner-Rasheed sarcoma viral oncogene homolog (FGR). In addition, a total of 17 upregulated genes, including the TPase family, AAA domain containing 2 (ATAD2) and cyclin-dependent kinase $4(C D K 4)$, exhibited copy number gains, while 5 downregulated genes, including Rho GTPase activating protein 9 (ARHGAP9) and major histocompatibility complex, class II, DO $\alpha$ (HLA-DOA), exhibited copy number losses. These results indicate that hyper-methylation of FCERIG, LEP, and FGR may serve a crucial function in the development of OS. In addition, copy number alterations of these DEGs, including ATAD2, CDK4, ARHGAP9 and $H L A-D O A$, may also contribute to OS progression. These DEGs may be candidate targets for the diagnosis and treatment of this disease.
\end{abstract}

Correspondence to: Dr Yong Ni, Department of Spinal Surgery, Shandong Jining No. 1 People's Hospital, 6 Jiankang Road, Jining, Shandong 272011, P.R. China

E-mail: doorld@163.com

Key words: osteosarcoma, differentially expressed genes, methylation, copy number analysis, functional enrichment analysis

\section{Introduction}

Osteosarcoma (OS) is the most common type of primary bone malignancy in children and adolescents (1). It is a high-grade malignant tumor with a poor prognosis, and $20 \%$ of patients with OS present with metastases at diagnosis $(2,3)$. Treatment generally involves surgery and adjuvant chemotherapy, and a positive response to chemotherapy is considered to be a prognostic marker for OS, indicating a favorable overall outcome $(4,5)$. Although neoadjuvant chemotherapy has improved the survival rate by a considerable amount, progress in treatment regimens remains at a plateau.

At the molecular level, OS is characterized by gross changes in gene expression and highly heterogeneous karyotypes with variable genomic aberrations (6-8). DNA methylation, particularly at cytosine-phosphate-guanine sites in the promoter region of genes, is a crucial mechanism for the downregulation of gene expression $(9,10)$. Expression levels of Ras association domain family member $1 \mathrm{~A}$ have been demonstrated to decrease due to promoter methylation in primary OS cell lines $(11,12)$. Hypermethylation of the hypermethylated in cancer 1 promoter has been revealed to be present in $17 \%$ of pediatric patients with OS (13). In addition, genetic variations, particularly single nucleotide polymorphisms, may contribute to cancer risk and progression (14). Copy number variations across the whole genome, including deletions, amplifications and duplications, are associated with OS tumorigenesis (15). In addition, the mutation or loss of gene expression of the tumor protein p53 tumor suppressor gene is commonly associated with OS $(16,17)$. Despite these data, there is limited understanding of the molecular pathogenesis of OS, and a lack of good diagnostic and prognostic tools.

A number of previous studies have combined gene expression data with DNA copy number data to screen for tumor-associated genes in OS genetic variants $(18,19)$. The microarray data GSE36004 has been used in the integrative analysis of mRNA expression, DNA methylation and DNA copy number in OS for elucidating dependencies, and the association between genetic and epigenetic alterations in OS (20). In contrast to a previous study (20), the present study aimed to utilize an integrative bioinformatics approach to map the genetic and epigenetic changes in OS, and to identify key genes associated with OS oncogenesis. Microarray data from 19 OS cell lines and normal controls was used to screen differentially expressed genes (DEGs) and differentially methylated regions (DMRs), and to 
perform copy number analysis. Then, functional enrichment analysis was performed and protein-protein interactions (PPI) were identified to additionally screen for the key genes. The identification of molecular targets that are specific for OS will be critical to the development of novel targeted therapeutic strategies to improve patient outcomes.

\section{Materials and methods}

Microarray data. The microarray data GSE36004, which was contributed by Kresse et al (20), were downloaded from the public repository Gene Expression Omnibus (GEO, http://www.ncbi.nlm.nih.gov/geo/) (21). It contained expression profiling data, methylation profiling data and genome variation single nucleotide polymorphism (SNP) profiling data, which were respectively based on Illumina HumanMethylation27 BeadChip (Illumina, Inc., San Diego, CA, USA), Affymetrix Genome-Wide Human SNP 6.0 Array (Affymetrix, Inc., Santa Clara, CA, USA) and Illumina human-6 v2.0 expression BeadChip (Illumina, Inc.) analyses. A total of 25 samples, including 19 OS cell lines and 6 normal controls (osteoblasts and bones), were applied to develop expression profiling data and methylation profiling data, respectively, while only the 19 OS cell lines were utilized to develop SNP profiling data. All the raw data and annotation files were obtained for subsequent analysis.

$D E G$ screening. According to the expression profiling data of GSE36004,DEGs in OS cell lines compared with normal controls were identified using the Limma package (available athttp://www. bioconductor.org/packages/release/bioc/html/limma.html) (22) in Bioconductor package version 1.0.2 (23). Significant P-values were adjusted for multiple testing using the Benjamini-Hochberg method (24). A log fold-change (FC) $>1$ and adjusted $\mathrm{P}<0.05$ were considered to indicate a statistically significant difference. Unsupervised hierarchical clustering of 25 samples was performed using the R package (v2.13.0; R Project for Statistical Computing, Vienna, Austria) (25) based on microarray data.

Analysis of methylated regions. Quantile normalization of the methylation profiling data was first performed using the Lumi package (available at http://www.bioconductor. org/packages/release/bioc/html/lumi.html) (26) in Bioconductor package version 1.0.2, then DMRs were identified using methyAnalysis (27) with minimum P-values adjusted to $<0.01$.

Copy number analysis. Copy number analysis of the raw data from the SNP profiling data was performed using the crlmm package (http://www.Bioconductor.org/packages/release/bioc/html/crlmm.html) (28) in Bioconductor package version 1.0.2. Then, genes with copy number gains/losses were additionally screened using the DNAcopy (29) and cghMCR package (http://www.bioconductor.org/packages $/ 2.4 / \mathrm{bioc} / \mathrm{html} / \mathrm{cghMCR} . \mathrm{html}$ ). Genes with copy number gains/losses ( 0.2 for gain, -0.2 for loss) in $>30 \%$ samples was the threshold value for the DNAcopy and cghMCR packages.

Functional enrichment analysis of DEGs. Gene Ontology (GO; http://www.geneontology.org) (30) is a tool for the unification of biology functions based on gene annotation information, which primarily consists of biological process (BP), molecular function (MF), and cellular component (CC) analysis. The Kyoto Encyclopedia of Genes and Genomes (KEGG; available at http://www.genome. ad.jp/kegg/) (31) is a pathway-associated database which connects known information on molecular interaction networks. To understand the biological significance of the identified DEGs, the upregulated and downregulated genes were input into the Database for Annotation Visualization and Integrated Discovery (DAVID; http://david.abcc.ncifcrf. gov/) (32) for GO BP terms and KEGG pathway analyses. $\mathrm{P}<0.05$ and gene counts $>2$ were considered to indicate a statistically significant difference.

PPI network construction. The Search Tool for the Retrieval of Interacting Genes database (STRING; http://www.bork .embl-heidelberg.de/STRING/) (33) provides comprehensive information on the functional interactions between DEGs and other genes by calculating their combined score. PPI pairs with a combined score of $>0.7$ as a cutoff value were identified. PPI networks were then constructed using Cytoscape software (version 2.6.3) (available at http://cytoscapeweb.cytoscape. org/) (34) based on the PPI pairs.

Statistical analysis. The correlation between the expression levels and the methylation levels of genes was analyzed by Pearson correlation coefficient test (35) using SPSS 19.0 software (IBM Corp., Armonk, NY, USA). The correlation coefficient takes a value between -1 and 1 , where the value closer to -1 (or 1) indicates a negative (or positive) high association and closer to 0 indicates no association between the two variables.

\section{Results}

DEG screening. A total of 663 DEGs with $\log \mathrm{FC}>1$ and adjusted $\mathrm{P}<0.05$ were screened using the Limma package of $\mathrm{R}$, including 227 upregulated and 436 downregulated DEGs. The resultant heat map of DEGs is depicted in Fig. 1.

Analysis of methylated regions. A total of 2,368 DMRs were identified using methyAnalysis with minimum P-values adjusted to <0.01, and 1,093 hyper- and 162 hypo-methylated regions were located in the gene promoter regions. Notably, there were 47 downregulated genes in the 1,093 hyper-methylated regions, while no genes were upregulated in the 162 hypo-methylated regions. Pearson correlation coefficient tests (35) was then used to calculate the correlation between the gene expression levels and the methylation levels of 47 downregulated genes. The correlation results demonstrated that the median, minimum and maximum values of the Pearson correlation coefficient were $-0.4987,-0.7902$ and -0.1573 , respectively. The genes with the strongest correlations were intercellular adhesion molecule $3(\mathrm{r}=-0.7902)$, cytochrome $\mathrm{c}$ oxidase subunit VIIa polypeptide $1(\mathrm{r}=-0.7743)$, aminomethyltransferase $(\mathrm{r}=-0.7554)$, retinoic acid receptor responder (tazarotene induced) $1(\mathrm{r}=-0.7517)$ and $\mathrm{Fc}$ fragment of $\mathrm{IgE}$, high affinity I, receptor for; gamma polypeptide (FCERIG; $r=-0.7366)$. 

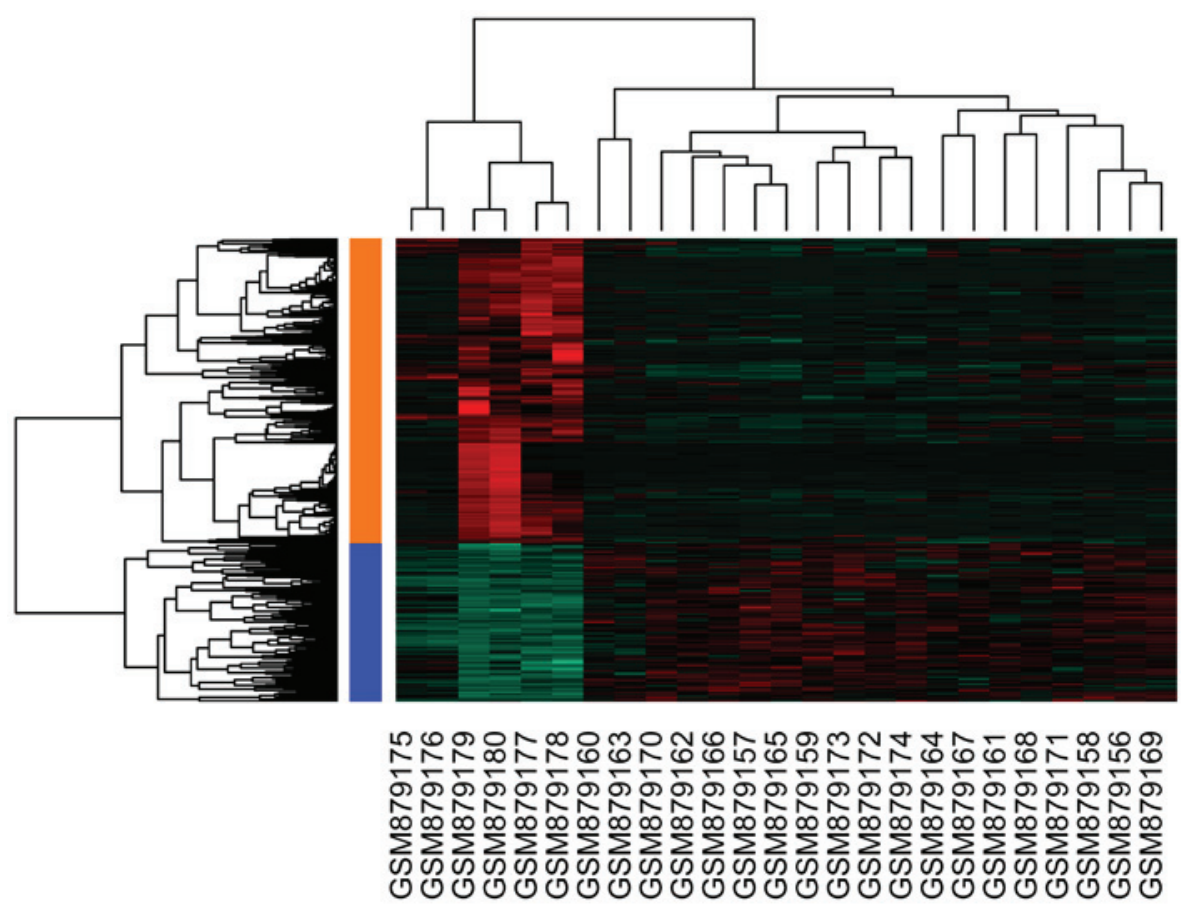

Figure 1. Heat map of DEGs. Samples with the serial numbers GSM879175-GSM879180 represent normal controls, and the others represent OS cell lines. The left vertical strips represent DEGs in OS cell lines compared with normal controls, with yellow strips representing downregulated genes and blue strips representing upregulated ones. DEGs, differentially expressed genes; OS, osteosarcoma.

Copy number analysis. Based on the microarray data for SNP profiling, 2,838 genes exhibited copy number gains and 415 exhibited copy number losses. Among them, 17 genes with copy number gains were upregulated, while 5 genes with copy number losses were downregulated. The genes with upregulated expression and copy number gains were as follows: ATPase family, AAA domain containing 2 (ATAD2), small nuclear ribonucleoprotein polypeptide $\mathrm{A}^{\prime}$, ribosomal protein $\mathrm{L} 7$ (RPL7), cyclin-dependent kinase $4(C D K 4)$ and tubulin, $\alpha$ la (TUBA1A). The genes with downregulated expression and copy number losses were Rho GTPase activating protein 9 (ARHGAP9), chromosome 1 open reading frame 54, leiomodin 3 (fetal), major histocompatibility complex, class II, DO $\alpha(H L A-D O A)$ and Rh blood group, D antigen (RHD).

Functional enrichment analysis of DEGs. DAVID was used to perform functional enrichment analysis of the DEGs. The over-represented GO BP terms and significantly enriched KEGG pathways are summarized in Tables I and II, respectively. According to the enrichment results, the upregulated genes were mainly associated with carboxylic acid biosynthetic, organic acid biosynthetic and L-serine metabolic processes (Table I). In addition, the upregulated genes were significantly enriched in 6 KEGG pathways, including glycine, serine and threonine metabolism, ribosome and one carbon pool by folate (Table II). Downregulated genes were mainly associated with functions including the immune response, defense response and gas transport (Table I), and were significantly enriched in 20 KEGG pathways, including asthma, viral myocarditis and intestinal immune network for immunoglobulin (Ig) A production (Table II).

Notably, the enrichment results demonstrated that 47 downregulated genes in the hyper-methylated regions were significantly enriched in the ECM-receptor interaction pathway, and were associated with the regulation of cytokine production, regulation of tumor necrosis factor production and cell adhesion.

PPI network analysis. Based on the information from the STRING database, PPI networks of upregulated and downregulated genes were constructed. MAD2 mitotic arrest deficient-like 1 (degree=9), BUB1 mitotic checkpoint serine/threonine kinase (degree $=8$ ) and PDZ binding kinase (degree=7) were selected as the hub nodes in the PPI network of upregulated genes (Fig. 2). Notably, the upregulated genes, including ATAD2, RPL7 and CDK4, exhibited copy number gains and may interact with other upregulated genes (Fig. 2).

In the PPI network of downregulated genes, TYRO protein tyrosine kinase binding protein (degree=37), immunoglobulin superfamily, member $6($ degree $=20)$ and lysosomal protein transmembrane 5 (degree=19) with the highest degrees were hub nodes (Fig. 3). In addition, the hyper-methylated hub nodes were FCERI G, leptin ( $L E P$ ), and feline Gardner-Rasheed sarcoma viral oncogene homolog $(F G R)$. Concurrently, ARHGAP9, HLA-DOA and RHD exhibited copy number losses and may interact with other downregulated genes.

\section{Discussion}

The cell line panel provides a valuable model system for analysis of genetic and epigenetic aberrations in OS (36). In the present study, a comprehensive bioinformatics approach was utilized for analysis of the effects of genome-wide changes in gene expression, DNA methylation and DNA copy number alterations in OS cell lines compared with the normal controls. The results demonstrated that a total of 
Table I. The top $10 \mathrm{GO}$ terms enriched among differentially expressed genes.

\begin{tabular}{|c|c|c|c|}
\hline GO ID & Description & Counts & P-value \\
\hline \multicolumn{4}{|l|}{ Upregulated } \\
\hline GO:0046394 & Carboxylic acid biosynthetic process & 13 & $1.33 \times 10^{-8}$ \\
\hline GO:0016053 & Organic acid biosynthetic process & 13 & $1.33 \times 10^{-8}$ \\
\hline GO:0006563 & L-serine metabolic process & 5 & $4.15 \times 10^{-7}$ \\
\hline GO:0008652 & Cellular amino acid biosynthetic process & 7 & $5.86 \times 10^{-6}$ \\
\hline GO:0006412 & Translation & 13 & $4.05 \times 10^{-5}$ \\
\hline GO:0009069 & Serine family amino acid metabolic process & 5 & $7.82 \times 10^{-5}$ \\
\hline GO:0009309 & Amine biosynthetic process & 7 & $8.49 \times 10^{-5}$ \\
\hline GO:0009070 & Serine family amino acid biosynthetic process & 4 & $1.09 \times 10^{-4}$ \\
\hline GO:0006564 & L-serine biosynthetic process & 3 & $2.37 \times 10^{-4}$ \\
\hline GO:0006633 & Fatty acid biosynthetic process & 6 & $7.00 \times 10^{-4}$ \\
\hline \multicolumn{4}{|l|}{ Downregulated } \\
\hline GO:0006955 & Immune response & 68 & $9.91 \times 10^{-24}$ \\
\hline GO:0006952 & Defense response & 52 & $3.80 \times 10^{-15}$ \\
\hline GO:0015669 & Gas transport & 9 & $3.36 \times 10^{-9}$ \\
\hline GO:0009611 & Response to wounding & 38 & $4.05 \times 10^{-9}$ \\
\hline GO:0032101 & Regulation of response to external stimulus & 20 & $7.43 \times 10^{-9}$ \\
\hline GO:0048584 & Positive regulation of response to stimulus & 23 & $4.88 \times 10^{-8}$ \\
\hline GO:0002504 & $\begin{array}{l}\text { Antigen processing and presentation of peptide or } \\
\text { polysaccharide antigen via MHC class II }\end{array}$ & 10 & $5.12 \times 10^{-8}$ \\
\hline GO:0002684 & Positive regulation of immune system process & 23 & $5.67 \times 10^{-8}$ \\
\hline GO:0050727 & Regulation of inflammatory response & 13 & $2.14 \times 10^{-7}$ \\
\hline GO:0006954 & Inflammatory response & 26 & $2.54 \times 10^{-7}$ \\
\hline
\end{tabular}

GO ID represents the identification number of the enriched GO term. Description represents the name of the GO term. Counts represent the number of upregulated or downregulated target genes. GO, gene ontology.

Table II. The significant KEGG pathways enriched by differentially expressed genes.

\begin{tabular}{|c|c|c|c|}
\hline Description & Counts & $\%$ & P-value \\
\hline \multicolumn{4}{|l|}{ Upregulated } \\
\hline hsa00260: Glycine, serine and threonine metabolism & 5 & 3.125 & $6.26 \times 10^{-04}$ \\
\hline hsa03010: Ribosome & 6 & 3.75 & 0.005144 \\
\hline hsa00670: One carbon pool by folate & 3 & 1.875 & 0.017709 \\
\hline hsa00100: Steroid biosynthesis & 3 & 1.875 & 0.019904 \\
\hline hsa01040: Biosynthesis of unsaturated fatty acids & 3 & 1.875 & 0.032434 \\
\hline hsa00450: Selenoamino acid metabolism & 3 & 1.875 & 0.04415 \\
\hline \multicolumn{4}{|l|}{ Downregulated } \\
\hline hsa05310: Asthma & 11 & 2.820513 & $9.14 \times 10^{-09}$ \\
\hline hsa05416: Viral myocarditis & 13 & 3.333333 & $1.71 \times 10^{-06}$ \\
\hline hsa04672: Intestinal immune network for IgA production & 11 & 2.820513 & $2.17 \times 10^{-06}$ \\
\hline hsa05330: Allograft rejection & 9 & 2.307692 & $1.21 \times 10^{-05}$ \\
\hline hsa04514: Cell adhesion molecules (CAMs) & 16 & 4.102564 & $1.45 \times 10^{-05}$ \\
\hline hsa05332: Graft-versus-host disease & 9 & 2.307692 & $2.26 \times 10^{-05}$ \\
\hline hsa04940: Type I diabetes mellitus & 9 & 2.307692 & $4.00 \times 10^{-05}$ \\
\hline hsa05322: Systemic lupus erythematosus & 13 & 3.333333 & $5.72 \times 10^{-05}$ \\
\hline hsa04640: Hematopoietic cell lineage & 12 & 3.076923 & $7.14 \times 10^{-05}$ \\
\hline hsa05320: Autoimmune thyroid disease & 9 & 2.307692 & $1.69 \times 10^{-04}$ \\
\hline
\end{tabular}

Description represents the identification number and the name of the KEGG pathway. Counts represent the number of upregulated or downregulated target genes. KEGG, Kyoto Encyclopedia of Genes and Genomes. 

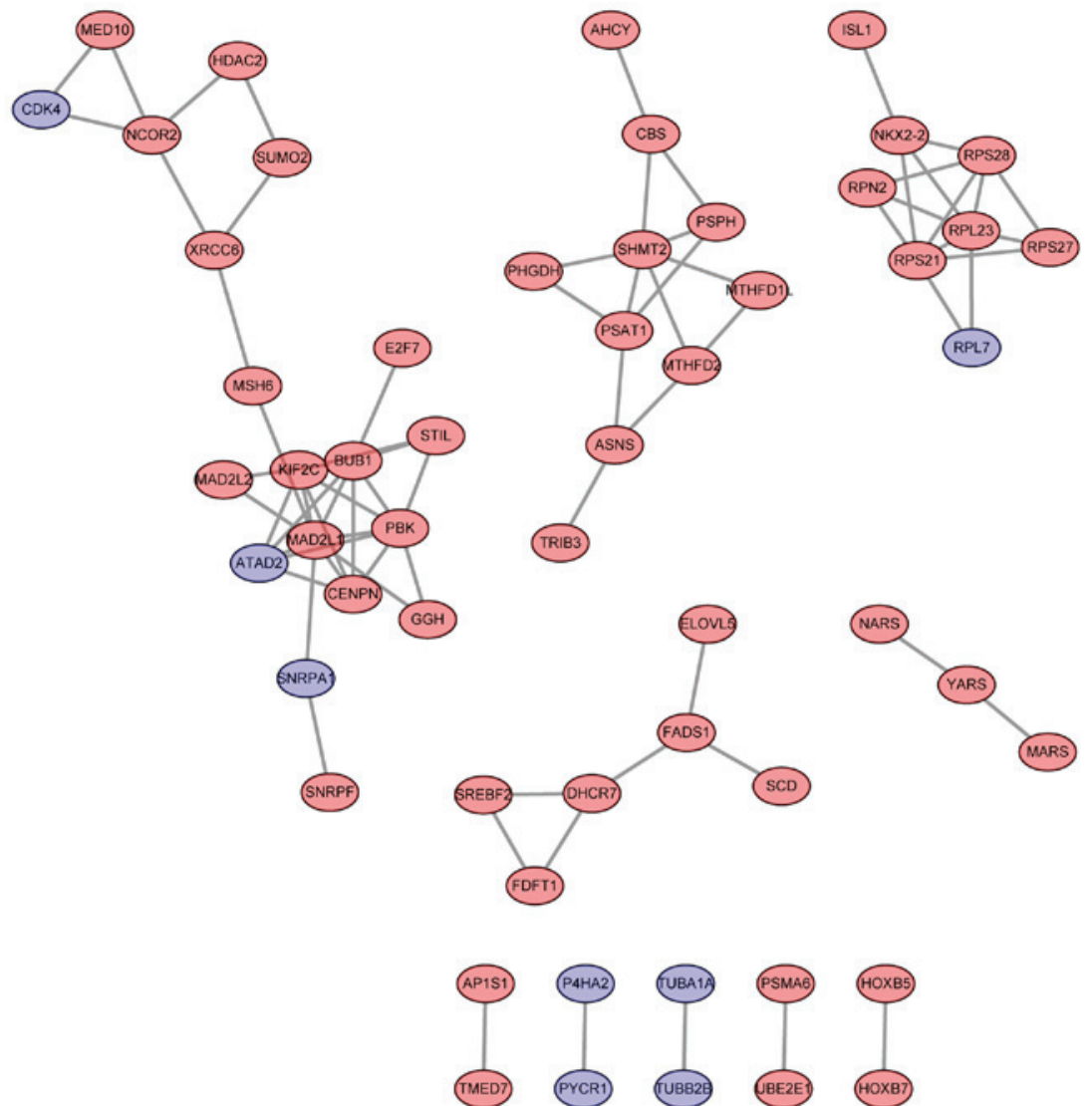

Figure 2. Protein-protein interaction network of upregulated genes. Red nodes represent upregulated genes. Blue nodes represent upregulated genes with copy number gain.
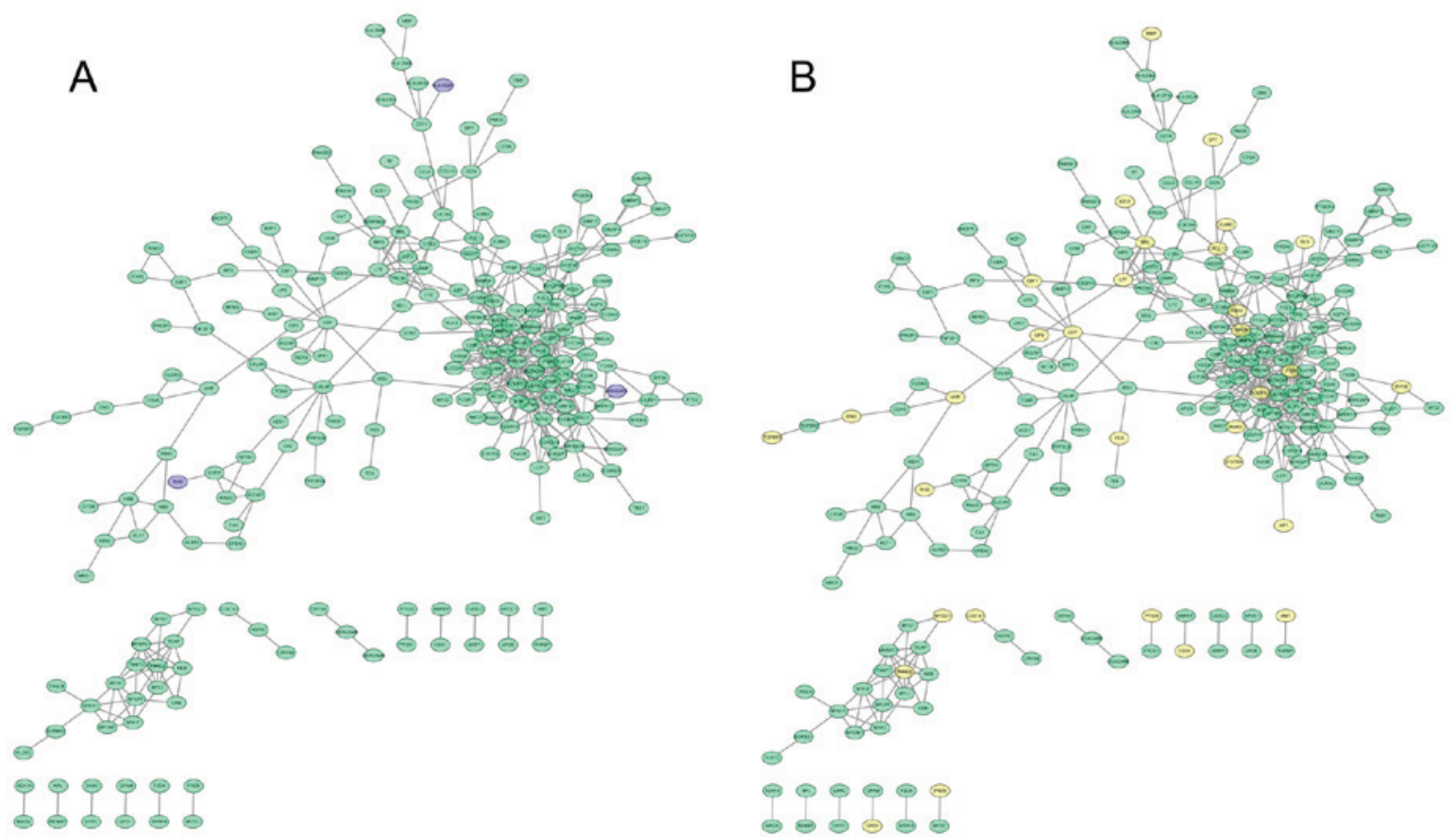

Figure 3. Protein-protein interaction network of downregulated genes. Green nodes represent downregulated genes. (A) Blue nodes represent downregulated genes with copy number loss. (B) Yellow nodes represent hyper-methylated downregulated genes.

47 downregulated genes were located in hyper-methylated regions, while no genes were upregulated in hypo-methylated regions, including FCERIG, FGR and LEP. In addition, downregulated genes including ARHGAP9 and HLA-DOA, exhibited copy number losses, and upregulated genes, including ATAD2 and CDK4, exhibited copy number gains. 
All these genes may act a key function in OS progression and merit additional discussion.

FCER $1 G, L E P$ and FGR were hyper-methylated in the present study. FCER1G, the $\gamma$ subunit gene of the high-affinity receptor for $\operatorname{IgE}(\mathrm{Fcc} R \mathrm{R})$, is considered to serve a crucial function in upregulating FceRI on atopic antigen-presenting cells (37). Demethylation of the FCERIG promoter results in the overexpression of FceRI on monocytes of patients with atopic dermatitis (38). Epigenetic deregulation may serve an important function in cancer development (39). LEP encodes the leptin protein which is expressed in and secreted from mature primary cultures of human osteoblasts, which is of importance for osteoblastic cell growth and bone mineralization (40). LEP may increase bone mass by stimulating OS cell proliferation via activation of the phosphoinositide 3-kinase and mitogen-activated protein kinase (MAPK) signaling pathways (41). The FGR gene is a member of the Src family of protein tyrosine kinases. This gene functions as a negative regulator of cell migration and adhesion, and is triggered by the $\beta$-2 integrin signal transduction pathway (42). The chemokine receptor 4/stromal cell-derived factor 1 serves an important function in OS tumor progression via the regulation of cell migration and adhesion (43), suggesting that FGR may be involved in OS development via regulating cell migration and adhesion. In the present study, these hyper-methylated genes were revealed to be downregulated, thus, it was hypothesized that these genes may be involved in OS progression via decreased expression following hyper-methylation. The results may assist in understanding the epigenetic regulation of specific genes in OS tumor development.

In addition, OS is a copy number alteration-rich malignant bone tumor, and candidate genes with copy number changes are being identified in OS (44). In the present study, DEGs including $A R H G A P 9$ and $H L A-D O A$, exhibited copy number losses while $A T A D 2$ and $C D K 4$ exhibited copy number gains. ARHGAP9, which is a novel MAP kinase docking protein, interacts with mitogen-activated protein kinase 1 (ERK2) and p38 $\alpha$ (45). Silencing ERK2 in human U2OS cells may inhibit the expression and function of glycoprotein 130, which serves a pivotal function in cancer and inflammation (46). In addition, $H L A-D O A$ is a key molecule in the antigen processing and presentation pathway, and this pathway has also been suggested to be involved in OS progression via downregulated expression of $H L A-D O A(47,48)$. In addition, ATAD2 is highly expressed and genetically amplified in several types of human cancer (49). ATAD2 binds to the v-Myc avian myelocytomatosis viral oncogene homolog $(c-M y c)$ oncogene and stimulates its transcriptional activity (49). A previous study demonstrated that overexpression of $c-M y c$ may promote OS cell invasion via the activation of the mitogen activated protein kinase kinase-extracellular signal-regulated kinase pathway (50). ATAD2 is also confirmed to exhibit prognostic significance in high-grade OS (51). The amplification and overexpression of $C D K 4$ tends to be associated with improved prognosis in low-grade OS (52). 12q13-14 CDK4 amplicons are frequently observed in OS (53). CDK4 and other CDK inhibitors are regarded as promising anticancer agents in cancer treatment (54). Therefore, these DEGs may serve important functions in the development and progression of OS. The results of the present study are consistent with these data, suggesting that copy number alterations of key genes may be associated with OS.

In conclusion, hyper-methylation of FCERIG, LEP and $F G R$ is observed in OS, suggesting that epigenetic alterations of these specific genes may act crucial functions in OS development. In addition, copy number alterations of these DEGs, including ARHGAP9, HLA-DOA, ATAD2 and CDK4, may also contribute to OS progression. These results indicate that genetic and epigenetic alterations are important mechanisms involved in OS, and these DEGs may serve as candidate targets for the diagnosis and treatment of this disease. However, no experimental validation and the relatively small sample size are the limitations of the present study. The results require additional validation.

\section{References}

1. Ottaviani G and Jaffe N: The epidemiology of osteosarcoma. In: Pediatric and Adolescent Osteosarcoma. Jaffe N, Bruland OS and Bielack S (eds). Springer, New York, pp3-13, 2010.

2. Posthuma De Boer J, Witlox MA, Kaspers GJ and van Royen BJ: Molecular alterations as target for therapy in metastatic osteosarcoma: A review of literature. Clin Exp Metastasis 28: 493-503, 2011.

3. Aksnes LH, Hall KS, Folleraas G, Stenwig AE, Bjerkehagen B, Taksdal I, Winderen M, Bruland OS and Saeter G: Management of high-grade bone sarcomas over two decades: The Norwegian Radium Hospital experience. Acta Oncol 45: 38-46, 2006.

4. Sakamoto A and Iwamoto Y: Current status and perspectives regarding the treatment of osteosarcoma: Chemotherapy. Rev Recent Clin Trials 3: 228-231, 2008.

5. Sadikovic B, Thorner P, Chilton-MacNeill S, Martin JW, Cervigne NK, Squire J and Zielenska M: Expression analysis of genes associated with human osteosarcoma tumors shows correlation of RUNX2 overexpression with poor response to chemotherapy. Bmc Cancer 10: 202, 2010.

6. SmidaJ,Baumhoer D, Rosemann M, Walch A, Bielack S, PorembaC, Remberger K, Korsching E, Scheurlen W, Dierkes C, et al: Genomic alterations and allelic imbalances are strong prognostic predictors in osteosarcoma. Clin Cancer Res 16: 4256-4267, 2010.

7. Squire JA, Pei J, Marrano P, Beheshti B, Bayani J, Lim G, Moldovan L and Zielenska M: High-resolution mapping of amplifications and deletions in pediatric osteosarcoma by use of CGH analysis of cDNA microarrays. Genes Chromosomes Cancer 38: 215-225, 2003.

8. Sandberg AA and Bridge JA: Updates on the cytogenetics and molecular genetics of bone and soft tissue tumors: Osteosarcoma and related tumors. Cancer Genet Cytogenet 145: 1-30, 2003.

9. Cui J, Wang W, Li Z, Zhang Z, Wu B and Zeng L: Epigenetic changes in osteosarcoma. Bull Cancer 98: E62-E68, 2011.

10. Momparler RL and Bovenzi V: DNA methylation and cancer. J Cell Physiol 183: 145-154, 2000.

11. Hou P, Ji M, Yang B, Chen Z, Qiu J, Shi X and Lu Z: Quantitative analysis of promoter hypermethylation in multiple genes in osteosarcoma. Cancer 106: 1602-1609, 2006.

12. Lim S, Yang MH, Park JH, Nojima T, Hashimoto H, Unni KK and Park YK: Inactivation of the RASSF1A in osteosarcoma. Oncol Rep 10: 897-901, 2003.

13. Rathi A, Virmani AK, Harada K, Timmons CF, Miyajima K, Hay RJ, Mastrangelo D, Maitra A, Tomlinson GE and Gazdar AF: Aberrant methylation of the HIC1 promoter is a frequent event in specific pediatric neoplasms. Clin Cancer Res 9: 3674-3678, 2003.

14. Batra J, Srinivasan S and Clements J: Single Nucleotide Polymorphisms (SNPs). In: Molecular Testing in Cancer. Springer, Yousef GM and Jothy S (eds). Springer Science and Buisiness Media, New York, NY, pp 55-80, 2014.

15. Gokgoz N, Wunder JS and Andrulis IL: Abstract 5075: Genome-wide analysis of DNA copy number variations in osteosarcoma. Cancer Res 72: 5075, 2012.

16. Seidinger AL, Mastellaro MJ, Paschoal Fortes F, Godoy Assumpção J, Aparecida Cardinalli I, Aparecida Ganazza M, Correa Ribeiro R, Brandalise SR, Dos Santos Aguiar S and Yunes JA: Association of the highly prevalent TP53 R337H mutation with pediatric choroid plexus carcinoma and osteosarcoma in southeast Brazil. Cancer 117: 2228-2235, 2011. 
17. Fu HL, Shao L, Wang Q, Jia T, Li M and Yang DP: A systematic review of p53 as a biomarker of survival in patients with osteosarcoma. Tumour Biol 34: 3817-3821, 2013.

18. Yen CC, Chen WM, Chen TH, Chen WY, Chen PC, Chiou HJ, Hung GY, Wu HT, Wei CJ, Shiau CY, et al: Identification of chromosomal aberrations associated with disease progression and a novel 3q13.31 deletion involving LSAMP gene in osteosarcoma. Int J Oncol 35: 775-788, 2009.

19. Lockwood WW, Stack D, Morris T, Grehan D, O'Keane C, Stewart GL, Cumiskey J, Lam WL, Squire JA, Thomas DM and O'Sullivan MJ: Cyclin E1 is amplified and overexpressed in osteosarcoma. J Mol Diagn 13: 289-296, 2011.

20. Kresse SH, Rydbeck H, Skårn M, Namløs HM, Barragan-Polania AH, Cleton-Jansen AM, Serra M, Liestøl K, Hogendoorn PC, Hovig E, et al: Integrative analysis reveals relationships of genetic and epigenetic alterations in osteosarcoma. PLoS One 7: e 48262, 2012

21. Barrett T, Wilhite SE, Ledoux P, Evangelista C, Kim IF, Tomashevsky M, Marshall KA, Phillippy KH, Sherman PM, Holko M, et al: NCBI GEO: Archive for functional genomics data sets-update. Nucleic Acids Res 41: D991-D995, 2013.

22. Smyth GK: Limma: Linear models for microarray data. In: Bioinformatics and Computational Biology Solutions Using R and Bioconductor. Gentleman R, Vincent JC, Huber W, Irizarry RA and Dudolt S (eds). Springer, pp 397-420, 2005.

23. Gentleman RC, Carey VJ, Bates DM, Bolstad B, Dettling M, Dudoit S, Ellis B, Gautier L, Ge Y, Gentry J, et al: Bioconductor: Open software development for computational biology and bioinformatics. Genome Biol 5: R80, 2004.

24. Benjamini Y and Hochberg Y: Controlling the false discovery rate: A practical and powerful approach to multiple testing. J Royal Stat Soc 57: 289-300, 1995.

25. R Core Team: R: A language and environment for statistical computing. R Foundation for Statistical Computing. Vienna, Austria, 2013.

26. Du P, Kibbe WA and Lin SM: Lumi: A pipeline for processing Illumina microarray. Bioinformatics 24: 1547-1548, 2008.

27. Du P and Bourgon R: methyAnalysis: An R package for DNA methylation data analysis and visualization. 2013.

28. Scharpf RB, Irizarry RA, Ritchie ME, Carvalho B and Ruczinski I: Using the $\mathrm{R}$ package crlmm for genotyping and copy number estimation. J Stat Softw 40: 1-32, 2011

29. Venkatraman E and Seshan A: DNAcopy: DNA copy number data analysis. R Package Version 1, 2010.

30. Ashburner M, Ball CA, Blake JA, Botstein D, Butler $\mathrm{H}$ Cherry JM, Davis AP, Dolinski K, Dwight SS, Eppig JT, et al: Gene ontology: Tool for the unification of biology. The Gene Ontology Consortium. Nat Genet 25: 25-29, 2000.

31. Kanehisa M and Goto S: KEGG: Kyoto encyclopedia of genes and genomes. Nucleic Acids Res 28: 27-30, 2000.

32. Huang DW, Sherman BT, Tan Q, Collins JR, Alvord WG, Roayaei J, Stephens R, Baseler MW, Lane HC and Lempicki RA: The DAVID gene functional classification tool: A novel biological module-centric algorithm to functionally analyze large gene lists. Genome Biol 8: R183, 2007.

33. Von Mering C, Huynen M, Jaeggi D, Schmidt S, Bork P and Snel B: STRING: A database of predicted functional associations between proteins. Nucleic Acids Res 31: 258-261, 2003.

34. Kohl M, Wiese S and Warscheid B: Cytoscape: Software for visualization and analysis of biological networks. Methods Mol Biol 696: 291-303, 2011.

35. Rahman $\mathrm{M}$ and Zhang Q: Comparison among pearson correlation coefficient tests. Far East J Math Sci (FJMS) 99: 237-255, 2016.

36. Mohseny AB, Machado I, Cai Y, Schaefer KL, Serra M, Hogendoorn PC, Llombart-Bosch A and Cleton-Jansen AM: Functional characterization of osteosarcoma cell lines provides representative models to study the human disease. Lab Invest 91: $1195-1205,2011$

37. Novak N, Tepel C, Koch S, Brix K, Bieber T and Kraft S: Evidence for a differential expression of the FcepsilonRIgamma chain in dendritic cells of atopic and nonatopic donors. J Clin Invest 111: 1047-1056, 2003.
38. Liang Y, Wang P, Zhao M, Liang G, Yin H, Zhang G, Wen $\mathrm{H}$ and Lu Q: Demethylation of the FCER1G promoter leads to FceRI overexpression on monocytes of patients with atopic dermatitis. Allergy 67: 424-430, 2012.

39. Ellis L, Atadja PW and Johnstone RW: Epigenetics in cancer: Targeting chromatin modifications. Mol Cancer Ther 8: 1409-1420, 2009.

40. Reseland JE, Syversen U, Bakke I, Qvigstad G, Eide LG, Hjertner O, Gordeladze JO and Drevon CA: Leptin is expressed in and secreted from primary cultures of human osteoblasts and promotes bone mineralization. J Bone Miner Res 16: 1426-1433, 2001.

41. Burguera B, Brunetto A, Garcia-Ocana A, Teijeiro R, Esplen J, Thomas T, Couce ME and Zhao A: Leptin increases proliferation of human steosarcoma cells through activation of PI(3)-K and MAPK pathways. Med Sci Monit 12: BR341-BR349, 2006.

42. Tronick SR, Popescu NC, Cheah MS, Swan DC, Amsbaugh SC, Lengel CR, DiPaolo JA and Robbins KC: Isolation and chromosomal localization of the human fgr protooncogene, a distinct member of the tyrosine kinase gene family. Proc Natl Acad Sci USA 82: 6595-6599, 1985.

43. Perissinotto E, Cavalloni G, Leone F, Fonsato V, Mitola S, Grignani G, Surrenti N, Sangiolo D, Bussolino F, Piacibello W and Aglietta M: Involvement of chemokine receptor 4/stromal cell-derived factor 1 system during osteosarcoma tumor progression. Clin Cancer Res 11: 490-497, 2005.

44. Pasic I, Shlien A, Durbin AD, Stavropoulos DJ, Baskin B, Ray PN, Novokmet A and Malkin D: Recurrent focal copy-number changes and loss of heterozygosity implicate two noncoding RNAs and one tumor suppressor gene at chromosome $3 \mathrm{q} 13.31$ in osteosarcoma. Cancer Res 70: 160-171, 2010.

45. Ang BK, Lim CY, Koh SS, Sivakumar N, Taib S, Lim KB, Ahmed S, Rajagopal G and Ong SH: ArhGAP9, a novel MAP kinase docking protein, inhibits Erk and p38 activation through WW domain binding. J Mol Signal 2: 1, 2007.

46. Bonito NA, Drechsler J, Stoecker S, Carmo CR, Seckl MJ, Hermanns HM and Costa-Pereira AP: Control of gp130 expression by the mitogen-activated protein kinase ERK2. Oncogene 33: 2255-2263, 2014

47. Luo $\mathrm{Y}$, Deng $\mathrm{Z}$ and Chen J: Pivotal regulatory network and genes in osteosarcoma. Arch Med Sci 9: 569-575, 2013.

48. Endo-Munoz L, Cumming A, Sommerville S, Dickinson I and Saunders NA: Osteosarcoma is characterised by reduced expression of markers of osteoclastogenesis and antigen presentation compared with normal bone. Br J Cancer 103: 73-81, 2010.

49. Ciró M, Prosperini E, Quarto M, Grazini U, Walfridsson J, McBlane F, Nucifero P, Pacchiana G, Capra M, Christensen J and Helin K: ATAD2 is a novel cofactor for MYC, overexpressed and amplified in aggressive tumors. Cancer Res 69: 8491-8498, 2009.

50. Swingler TE, Wheeler G, Carmont V, Elliott HR, Barter MJ, Abu-Elmagd M, Donell ST, Boot-Handford RP, Hajihosseini MK, Münsterberg A, et al: The expression and function of microRNAs in chondrogenesis and osteoarthritis. Arthritis Rheum 64: 1909-1919, 2012.

51. Fellenberg J, Bernd L, Delling G, Witte D and Zahlten-Hinguranage A: Prognostic significance of drug-regulated genes in high-grade osteosarcoma. Mod Pathol 20: 1085-1094, 2007.

52. Kyriazoglou AI, Vieira J, Dimitriadis E, Arnogiannaki N, Teixeira MR and Pandis N: 12q amplification defines a subtype of extraskeletal osteosarcoma with good prognosis that is the soft tissue homologue of parosteal osteosarcoma. Cancer Genet 205: 332-336, 2012.

53. Mejia-Guerrero S, Quejada M, Gokgoz N, Gill M, Parkes RK, Wunder JS and Andrulis IL: Characterization of the $12 \mathrm{q} 15$ MDM2 and 12q13-14 CDK4 amplicons and clinical correlations in osteosarcoma. Genes Chromosomes Cancer 49: 518-525, 2010.

54. Finn RS, Crown JP, Lang I, Boer K, Bondarenko IM, Kulyk SO, Ettl J, Patel R, Pinter T, Schmidt M, et al: Abstract S1-6: Results of a randomized phase 2 study of PD 0332991, a cyclin-dependent kinase (CDK) 4/6 inhibitor, in combination with letrozole vs. letrozole alone for first-line treatment of ER+/HER2-advanced breast cancer (BC). Cancer Res 72 (24 Suppl): S1-S6, 2012. 